\title{
Inertial effects in anomalous dielectric relaxation
}

\author{
W. T. Coffey, ${ }^{1}$ Yu. P. Kalmykov, ${ }^{2}$ and S. V. Titov ${ }^{3}$ \\ ${ }^{1}$ Department of Electronic and Electrical Engineering, School of Engineering, Trinity College, Dublin 2, Ireland \\ ${ }^{2}$ Centre d'Etudes Fondamentales, Université de Perpignan, 52, Avenue de Villeneuve, 66860 Perpignan Cedex, France \\ ${ }^{3}$ Institute of Radio Engineering and Electronics of the Russian Academy of Sciences, Vvedenskii Square 1, Fryazino, \\ Moscow Region 141190, Russia
}

(Received 9 October 2001; published 15 February 2002)

\begin{abstract}
The inertia corrected Debye model of rotational Brownian motion of polar molecules is generalized to fractional dynamics (anomalous diffusion) in the context of the fractional Klein-Kramers equation. The fractal generalization of the Gross-Sack solution for the complex dielectric susceptibility $\chi(\omega)$ for an assembly of fixed axis rotators is given. The high-frequency behavior of $\chi(\omega)$ is controlled by the inertia of a dipole as in normal diffusion, so that the Gordon sum rule for dipolar absorption is satisfied ensuring a return to optical transparency at very high frequencies.
\end{abstract}

DOI: 10.1103/PhysRevE.65.032102

PACS number(s): 05.40.Jc, 77.22.-d, 05.45.Df

Relaxation functions for fractal random walks are fundamental in the kinetics of complex systems such as liquid crystals, amorphous semiconductors and polymers, glass forming liquids, etc. [1]. Relaxation in these systems may deviate considerably from the exponential (Debye) pattern. An important task in dielectric relaxation of complex systems is to extend [2,3] the Debye theory of relaxation of polar molecules to fractional dynamics, so that empirical decay functions, e.g., the stretched exponential of Williams and Watts [4], may be justified in terms of fractal random walks.

A marked deficiency [5-10] of the Debye theory is that it cannot describe the dielectric relaxation process at short times [of the order of the characteristic decay time of the dipole angular velocity correlation function (AVCF)] or at high frequencies (HF) (in the far-infrared region) as like the Einstein theory of the translational Brownian motion [11] it is based on the Smoluchowski equation. That approximate equation describing the evolution of the probability density function (PDF) of the orientations of the dipoles in configuration space [12] is only valid when dipole inertial effects are negligible as it assumes instantaneous equilibrium of the angular velocities. Thus the Debye theory predicts infinite integral absorption.

The incorporation of such inertial effects in the Debye theory, ensuring a return to optical transparency at HF, was first achieved by Rocard [13] and a completely rigorous treatment was given by Gross [6] and Sack [7]. They used the Klein-Kramers equation applied to rotational Brownian motion, describing the evolution of the PDF in configuration-angular velocity space. Hence they obtained exact expressions for the complex susceptibility of noninteracting rigid dipoles as infinite continued fractions. The simple relaxation equation of Rocard [13] then appears for small inertial effects, i.e., where the ratio of the characteristic relaxation time of the AVCF $\tau_{\omega}$ to the (Debye) relaxation time $\tau$ of the dipole relaxation function is small.

Recently Barkai and Silbey [14] have proposed a generalization of the Klein-Kramers equation for the inertia corrected translational Brownian motion to fractional dynamics. Here we demonstrate how a similar fractional Klein-Kramers equation (FKKE) for the anomalous rotational diffusion may be solved using the generalized integration theorem [1] of Laplace transformation yielding the exact complex dielectric susceptibility in continued fraction form as in the normal Brownian motion [6,7]. The method may also be obviously extended to all problems where solutions of the KleinKramers or more generally $[12,15]$ the Fokker-Planck equation, may be expressed as recurrence relations involving ordinary or matrix continued fractions $[11,15]$.

We illustrate by considering the simplest microscopic model of dielectric relaxation, namely: an assembly of noninteracting rigid dipoles of moment $\mu$ each rotating about a fixed axis through its center [6,7]. (An assembly of fixed-axis rotators qualitatively reproduces the principal features of dielectric relaxation of dipolar molecules in space while allowing considerable mathematical simplification of the problem) $[6,7]$. A dipole has moment of inertia $I$ and is specified by the angular coordinate $\phi$ so that it constitutes a system of 1 (rotational) degree of freedom. The FKKE for the PDF $W(\phi, \dot{\phi}, t)$ in the space $(\phi, \dot{\phi})$ is identical to that of the onedimensional translational Brownian motion of a particle [15] however, rotational quantities (angle $\phi$, moment of inertia $I$, etc.) replace translational ones (position $x$, mass $m$, etc.) so that

$$
\frac{d W}{d t}=\frac{\partial W}{\partial t}+\dot{\phi} \frac{\partial W}{\partial \phi}-\frac{\mu E \sin \phi}{I} \frac{\partial W}{\partial \dot{\phi}}={ }_{0} D_{t}^{1-\alpha} L_{\mathrm{FP}} W
$$

where

$$
{ }_{0} D_{t}^{1-\alpha} L_{\mathrm{FP}} W={ }_{0} D_{t}^{1-\alpha} \vartheta \beta\left(\frac{\partial}{\partial \dot{\phi}}(\dot{\phi} W)+\frac{k_{B} T}{I} \frac{\partial^{2} W}{\partial \dot{\phi}^{2}}\right)
$$

is the fractional Fokker-Planck operator, $k_{B}$ is the Boltzmann constant, $T$ is the temperature, $\beta=\zeta / I, \zeta$ is the viscous damping coefficient of a dipole, $\vartheta=\tau^{1-\alpha}, \alpha$ is the anomalous exponent characterizing the fractal time process, and $\tau$ is the intertrapping time scale identified with the Debye relaxation time $\zeta / k_{B} T$ (at ambient temperatures, $\tau$ is of the order $10^{-11} \mathrm{sec}$ for molecular liquids and solutions). Thus the fractional dynamics emerges from the competition of 
Brownian motion events of average duration $\tau$ interrupted by trapping events whose duration is broadly distributed [1]. In writing Eq. (1) we assume that a weak uniform electric field $\mathbf{E}$ applied along the initial line is suddenly switched off at time $t=0$ so that linear response theory [10] may be used to describe the ensuing response. In the absence of $\mathbf{E}$, Eq. (1) describes anomalous diffusion; the value $\alpha=1$ corresponds to normal diffusion [14]. The operator ${ }_{0} D_{t}^{1-\alpha}$ $\equiv(\partial / \partial t){ }_{0} D_{t}^{-\alpha}$ in Eq. (1) is defined in terms of the convolution (the Riemann-Liouville fractional integral definition) [1],

$$
{ }_{0} D_{t}^{-\alpha} W(\phi, t)=\frac{1}{\Gamma(\alpha)} \int_{0}^{t} \frac{W\left(\phi, t^{\prime}\right) d t^{\prime}}{\left(t-t^{\prime}\right)^{1-\alpha}}
$$

so that the fractional derivative is a type of memory function $[1,5]$ or stosszahlansatz (collision integral) for the Boltzmann equation underlying the Fokker-Planck equation. We remark that a slowly-decaying power-law kernel in the Riemann-Liouville operator (2) is typical of memory effects in complex systems. In this context we remark that the operator ${ }_{0} D_{t}^{1-\alpha}$ in Eq. (1) does not act on $\dot{\phi}(\partial W / \partial \phi)$ and $-\mu E I^{-1} \sin \phi(\partial W / \partial \dot{\phi})$ (i.e., the Liouville or conservative terms) in the convective derivative $\dot{W}$ so that the conventional form of the Boltzmann equation with Fokker-Planck stosszahlansatz as modified by ${ }_{0} D_{t}^{1-\alpha}$ is preserved. If the fractional derivative acts on the convective term, i.e., the conservative part of Eq. (1), then nonphysical behavior (i.e., infinite integral absorption) of the dielectric absorption coefficient occurs as in the Debye theory of dielectric relaxation [5].

We may seek a solution of Eq. (1) as in normal diffusion as $[5,11]$

$$
W(\phi, \dot{\phi}, t)=e^{-\eta^{2} \dot{\phi}^{2}} \sum_{p=-\infty}^{\infty} \sum_{n=0}^{\infty} c_{p, n}(t) e^{i p \phi} H_{n}(\eta \dot{\phi}),
$$

where $\eta=\sqrt{I / 2 k_{B} T}$ and the $H_{n}(x)$ are the Hermite polynomials [16]. The linearized initial (at $t=0$ ) PDF is

$$
W(\phi, \dot{\phi}, 0) \approx \frac{1}{2 \pi^{3 / 2}} \eta e^{-\eta^{2} \dot{\phi}^{2}}\left[1+\frac{\mu E}{k_{B} T} \cos \phi\right] .
$$

Straightforward manipulation $[5,10,11]$ of the recurrence relations of the $H_{n}$ [16] leads by orthogonality, to a differential recurrence relation for the coefficients $c_{p, n}(t)$, which may be written [11] for the only case of interest (since the linear response is considered), namely, $p=1$, viz.,

$$
\begin{aligned}
& \frac{d}{d t} c_{1, n}(t)+\frac{i}{2 \eta}\left[2(n+1) c_{1, n+1}(t)+c_{1, n-1}(t)\right] \\
& \quad=-{ }_{0} D_{t}^{1-\alpha} \vartheta n \beta c_{1, n}(t) .
\end{aligned}
$$

On using the integration theorem of Laplace transformation as generalized to fractal calculus [1], viz.,

$$
L\left\{{ }_{0} D_{t}^{1-\alpha} f(t)\right\}=\left\{\begin{array}{l}
s^{1-\alpha} \widetilde{f}(s)-\left.D_{t}^{-\alpha} f(t)\right|_{t=0} \quad(0<\alpha<1), \\
s^{1-\alpha} \widetilde{f}(s) \quad(1 \leqslant \alpha<2),
\end{array}\right.
$$

where

$$
\widetilde{f}(s)=L\{f(t)\}=\int_{0}^{\infty} e^{-s t} f(t) d t,
$$

we have from Eq. (5),

$$
\begin{aligned}
{[2 \tau s+} & \left.n \gamma^{\prime 2}(\tau s)^{1-\alpha}\right] \widetilde{c}_{1, n}(s)+i \gamma^{\prime}\left[2(n+1) \widetilde{c}_{1, n+1}(s)\right. \\
& \left.+\widetilde{c}_{1, n-1}(s)\right]=c_{1, n}(0) .
\end{aligned}
$$

Here $\gamma^{\prime}=\tau / \eta=\zeta \sqrt{2 / I k_{B} T}$ is the inertial effects parameter. Noting the initial condition, Eq. (4), all the $c_{1, n}(0)$ in Eq. (6) will vanish with the exception $n=0$. Furthermore Eq. (6) is an example of how using the Laplace integration theorem above, all recurrence relations associated with the Brownian motion may be generalized to fractional dynamics. The normalized complex susceptibility $\hat{\chi}(\omega)=\hat{\chi}^{\prime}(\omega)-i \hat{\chi}^{\prime \prime}(\omega)$ is given by linear response theory as $[10]$

$$
\hat{\chi}(\omega)=\frac{\chi(\omega)}{\chi^{\prime}(0)}=1-i \omega \frac{\widetilde{c}_{1,0}(i \omega)}{c_{1,0}(0)},
$$

where $\chi^{\prime}(0)=\mu^{2} N /\left(2 k_{B} T\right)$ and $N$ is the number of dipoles per unit volume. [We remark that $\widetilde{c}_{1,0}(s)$ also yields the Laplace transform of the characteristic function of the configuration space PDF.]

Equation (6), which is a three term algebraic recurrence relation for the $\widetilde{c}_{1, n}(s)$, can be solved in terms of continued fractions [11] then yielding the generalization of the familiar Gross-Sack result $[6,7]$ for a fixed axis rotator to fractional relaxation, namely,

$\hat{\chi}(\omega)$

$$
=1-\frac{B(i \omega \tau)^{\sigma}}{B(i \omega \tau)^{\sigma}+\frac{B}{1+B(i \omega \tau)^{\sigma}+\frac{2 B}{2+B(i \omega \tau)^{\sigma}+\frac{3 B}{3+\cdots}}}},
$$

where $\sigma=2-\alpha$ and $B=2 \gamma^{\prime-2}(i \omega \tau)^{2(\alpha-1)}$. Equation (8), in turn, can be expressed in terms of the confluent hypergeometric (Kummer) function $M(a, b, z)[16]$, viz.,

$$
\hat{\chi}(\omega)=1-\frac{(i \tau \omega)^{\sigma}}{1+(i \tau \omega)^{\sigma}} M\left(1,1+B\left[1+(i \tau \omega)^{\sigma}\right], B\right) .
$$

Equation (9) can readily be derived by comparing Eq. (8) with the continued fraction

$$
\frac{M(a, b, z)}{(b-1) M(a-1, b-1, z)}=\frac{1}{b-1-z+\frac{a z}{b-z+\frac{(a+1) z}{b+1-z+\cdots}}},
$$




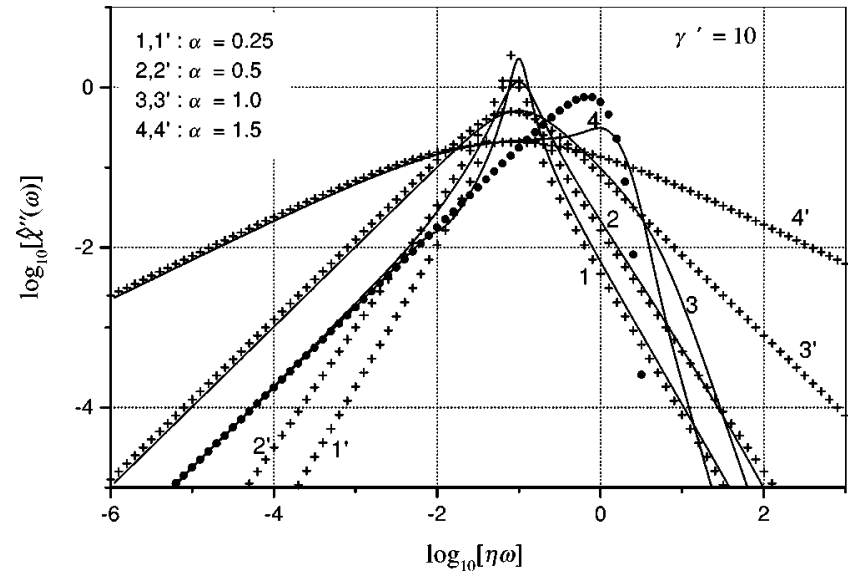

FIG. 1. Dielectric loss spectra $\hat{\chi}^{\prime \prime}(\omega)$ for $\gamma^{\prime}=10$ and various values of $\alpha: \alpha=0.25$ (curves 1 and $1^{\prime}$ ), $\alpha=0.5\left(2,2^{\prime}\right), \alpha=1$ (3, $\left.3^{\prime}\right)$, and $\alpha=1.5\left(4,4^{\prime}\right)$. Solid lines (1, 2, 3, and 4), Eq. (9); crosses $\left(1^{\prime}, 2^{\prime}, 3^{\prime}\right.$, and $\left.4^{\prime}\right)$, Eq. (12); filled circles, Eq. (14).

with $a=1, z=B$, and $b=1+B\left[1+(i \tau \omega)^{\sigma}\right]$ and by noting that $M(0, b-1, z)=1[16]$. The continued fraction (10) can be obtained from the known recurrence relation [16]

$$
\begin{aligned}
& b(1-b+z) M(a, b, z)-a z M(a+1, b+1, z)+b(b-1) \\
& \quad \times M(a-1, b-1, z)=0 .
\end{aligned}
$$

We remark that $M(1,1+b, z)=b z^{-b} e^{z} \gamma(b, z)$, where $\gamma(b, z)=\int_{0}^{z} e^{-t} t^{b-1} d t$ is the incomplete gamma function [16].

For $\sigma=1$, Eq. (9) can be reduced to Sack's result [Eq. (3.19c) of Ref. [7]]. In the high damping limit $\left(\gamma^{\prime} \gg 1\right)$, Eq. (9) can be simplified yielding the generalization $[3,4]$ to fractional dynamics of the Rocard $[9,13]$ equation, namely,

$$
\hat{\chi}(\omega)=\frac{1}{1+(i \omega \tau)^{\sigma}-2\left(\omega \tau / \gamma^{\prime}\right)^{2}} .
$$

On neglecting inertial effects $\left(\gamma^{\prime} \rightarrow \infty\right)$, Eq. (9) becomes

$$
\hat{\chi}(\omega)=1 /\left[1+(i \omega \tau)^{\sigma}\right],
$$

i.e., the result previously proposed from empirical considerations [4]. For $\sigma=1$, Eq. (12) reduces to the Debye equation.

Dielectric loss spectra $\hat{\chi}^{\prime \prime}(\omega)$ versus $\log _{10}(\omega \eta)$, for various values of $\alpha$ and $\gamma^{\prime}$ are shown in Figs. 1-3. It is apparent that the spectral parameters (the characteristic frequency, the half-width, the shape) strongly depend on both $\alpha$ (which pertains to the velocity space) and $\gamma^{\prime}$. Moreover the HF behavior of $\hat{\chi}^{\prime \prime}(\omega)$ is entirely determined by the inertia of system. It is apparent just as in Brownian dynamics that inertial effects produce a much more rapid fall off of $\hat{\chi}^{\prime \prime}(\omega)$ at HF. Thus the Gordon sum rule for the dipole integral absorption of one-dimensional rotators [5], viz.,

$$
\int_{0}^{\infty} \omega \chi^{\prime \prime}(\omega) d \omega=\left(\pi N \mu^{2}\right) /(4 I)
$$

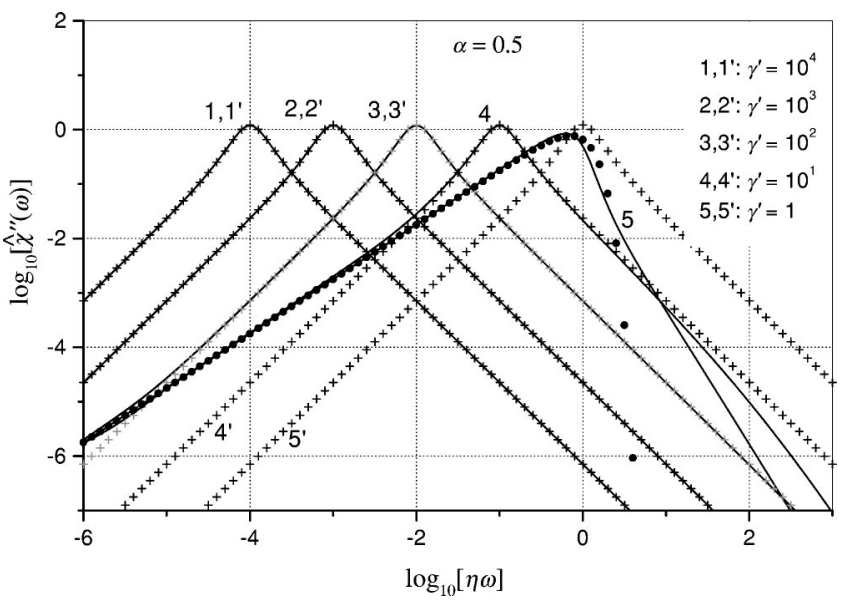

FIG. 2. Dielectric loss spectra $\hat{\chi}^{\prime \prime}(\omega)$ for $\alpha=0.5$ (enhanced diffusion) and various values of $\gamma^{\prime}: \gamma^{\prime}=10^{4}$ (curves 1 and $1^{\prime}$ ), $\gamma^{\prime}$ $=10^{3}\left(2,2^{\prime}\right), \gamma^{\prime}=10^{2}\left(3,3^{\prime}\right), \gamma^{\prime}=10\left(4,4^{\prime}\right)$, and $\gamma^{\prime}=1\left(5,5^{\prime}\right)$. Solid lines (1, 2, 3, 4, and 5), Eq. (9); crosses $\left(1^{\prime}, 2^{\prime}, 3^{\prime}, 4^{\prime}\right.$, and 5'), Eq. (12); filled circles, Eq. (14).

is satisfied. It is significant that the right-hand side of Eq. (13) is determined by molecular parameters only and does not depend on temperature. Such behavior is quite unlike that resulting from the hypothesis that the fractional derivative acts on the convective term leading always [17] to infinite integral absorption as in the Debye (excluding inertial effects) and Van Vleck-Weisskopf kinetic models $[6,7,18]$. Moreover, it is apparent from Figs. 1 and 3 assuming that Eq. (1) also describes subdiffusion in configuration space (that is, $\sigma<1$ or $2>\alpha>1$ ), then Eq. (9) also provides a physically acceptable description of the loss spectrum. Here, in the high damping limit $\left(\gamma^{\prime} \gg 1\right)$, the low-frequency part of $\hat{\chi}^{\prime \prime}(\omega)$ may be approximated by the modified Debye Eq. (12). For $\alpha<1$ corresponding to $\sigma>1$ (enhanced diffusion in configuration space), the low-frequency behavior of $\hat{\chi}^{\prime \prime}(\omega)$ is similar (see Figs. 1 and 3) to that of the dielectric loss $\hat{\chi}_{\mathrm{FR}}^{\prime \prime}(\omega)$ in the free rotation limit $(\zeta=0)$, which is given by $[6,7]$

$$
\hat{\chi}_{\mathrm{FR}}^{\prime \prime}(\omega)=\sqrt{\pi} \eta \omega e^{-\eta^{2} \omega^{2}} .
$$

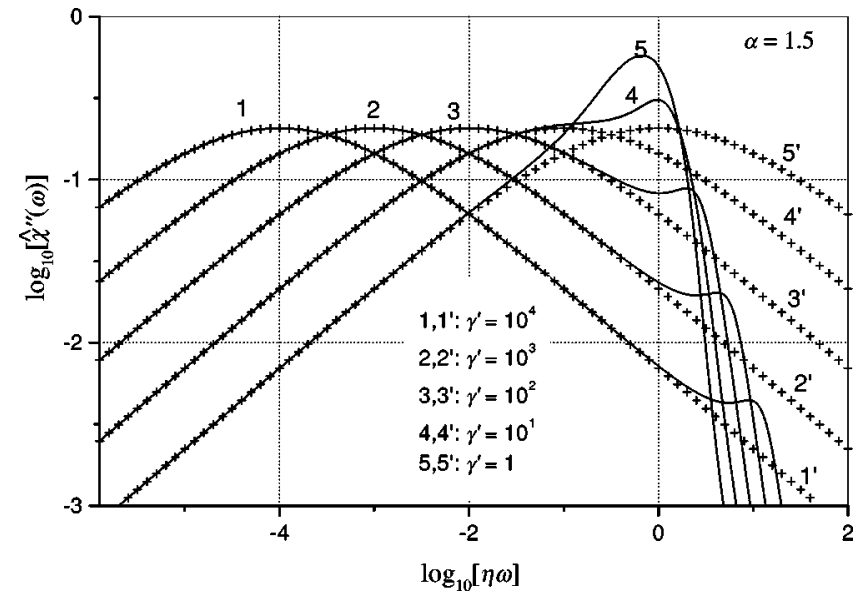

FIG. 3. The same as in Fig. 2 for $\alpha=1.5$ (subdiffusion). 
The HF dielectric absorption in the far-infrared region is proportional to the spectrum of $\langle\dot{\phi}(0) \dot{\phi}(t)\rangle_{0}$ and can be used to determine that AVCF experimentally [5]. In order to calculate the equilibrium AVCF $\langle\dot{\phi}(0) \dot{\phi}(t)\rangle_{0}$ in the fractional dynamics, one can simply adapt the result of Barkai and Silbey [14] for the translational $\operatorname{VCF}\langle\dot{x}(0) \dot{x}(t)\rangle_{0}$ replacing translational quantities by rotational ones. We have

$$
\langle\dot{\phi}(0) \dot{\phi}(t)\rangle_{0}=\frac{k_{B} T}{I} E_{\alpha}\left(-\frac{\gamma^{\prime 2}}{2}\left(\frac{t}{\tau}\right)^{\alpha}\right),
$$

where $E_{\alpha}(z)$ is the Mittag-Leffler function [1] defined by

$$
E_{\alpha}(z)=\sum_{n=0}^{\infty} \frac{z^{n}}{\Gamma(1+\alpha n)} \text {. }
$$

Equation (15) represents the generalization of the AVCF of the Ornstein-Uhlenbeck [14,19] (inertia corrected Einstein) theory of the Brownian motion to fractional dynamics. The long time tail due to the asymptotic $(t \gg \tau) t^{-\alpha}$ like dependence [1] of the $\langle\dot{\phi}(0) \dot{\phi}(t)\rangle_{0}$ is apparent, as is the stretched exponential behavior at short times $(t \ll \tau)$. For $\alpha>1$, $\langle\dot{\phi}(0) \dot{\phi}(t)\rangle_{0}$ exhibits oscillations (see Fig. 4) consistent with the large excess absorption occurring at HF.

In evaluating the dielectric response, we have ignored (i) quantum effects, (ii) dipole-dipole interactions, and (iii) the influence of the internal field [5]. The condition (i) means that our results are applicable when the inequality $\eta \hbar / I \ll 1$ ( $\hbar$ is the Planck constant) is fulfilled [6]. The above inequality breaks down in liquids only for the lightest polar molecules such as $\mathrm{HCl}$. The condition (ii) reflects the fact that dipole-dipole correlations are neglected in the FKEE Eq. (1), which is only valid in the zero order of the small parameter $\mu^{2} N_{0} / k T \ll 1$; the last inequality is satisfied at room temperatures and for dipole moments $\sim 10^{-18}$ CGS units up to a concentration of $10^{21} \mathrm{~cm}^{-3}$. This condition, as well as (iii) (i.e., the macroscopic, or Maxwell, field is equal to the applied electric field) is satisfied in dilute dipolar systems, e.g., solutions of polar molecules in nonpolar solvents. One may

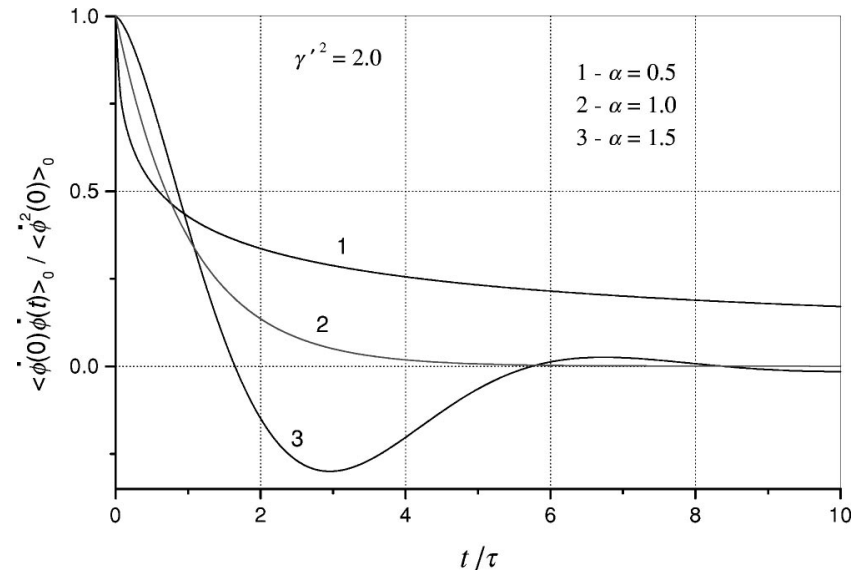

FIG. 4. The normalized angular velocity correlation function $\langle\dot{\phi}(0) \dot{\phi}(t)\rangle_{0} /\left\langle\dot{\phi}^{2}(0)\right\rangle_{0}$ for $\gamma^{\prime 2}=2$ and $\alpha=0.5$ (curve 1), $\alpha=1.0$ (curve 2), and $\alpha=1.5$ (curve 3 ).

expect, however, that the qualitative behavior of the dielectric response will be similar at higher concentrations of dipoles.

To conclude, we have demonstrated, how existing Brownian motion solutions [6,7] for dielectric relaxation may be generalized to fractional dynamics using continued fractions [11]. The result is of particular interest in dielectric relaxation as it demonstrates how the unphysical HF divergence of the absorption coefficient due to the neglect of inertia may be removed in fractional relaxation as in inertia corrected Debye relaxation (see Figs. 1-3). The methods we have outlined are also of interest when extended to other relaxation models such as the itinerant oscillator [20], which attempt to incorporate both resonance and relaxation behavior in a single model with the purpose of simultaneously explaining the Debye (low-frequency) and far infrared absorption spectra of complex dipole systems.

The support of this work by the Enterprise Ireland Research Collaboration Fund 2001, USAF, EOARD (Contract No. F61773-01-WE407) and the Russian Foundation for Basic Research (Project No. 01-02-16050) is gratefully acknowledged.
[1] R. Metzler and J. Klafter, Adv. Chem. Phys. 116, 223 (2001).

[2] W. G. Glöckle et al., J. Stat. Phys. 71, 741 (1993).

[3] K. Weron and M. Kotulski, Physica A 232, 180 (1996).

[4] G. Williams et al., Trans. Faraday Soc. 66, 80 (1970).

[5] M. W. Evans et al., Molecular Dynamics and Theory of Broadband Spectroscopy (Wiley, New York, 1982).

[6] E. P. Gross, J. Chem. Phys. 23, 1415 (1955).

[7] R. A. Sack, Proc. Phys. Soc. London, Sect. B 70, 402 (1957).

[8] J. R. McConnell, Rotational Brownian Motion and Dielectric Theory (Academic Press, New York, 1980).

[9] B. K. P. Scaife, Principles of Dielectrics (Oxford University Press, London, 1989).

[10] W. T. Coffey, M. W. Evans, and P. Grigolini, Molecular Diffusion and Spectra (Wiley, New York, 1984) [Russian edition (Mir, Moscow, 1987)].
[11] W. T. Coffey et al., The Langevin Equation (World Scientific, Singapore, 1996).

[12] W. T. Coffey et al., Adv. Chem. Phys. 117, 483 (2001).

[13] M. Y. Rocard, J. Phys. Radium 4, 247 (1933).

[14] E. Barkai and R. S. Silbey, J. Phys. Chem. B 104, 3866 (2000).

[15] Yu. P. Kalmykov et al., Phys. Rev. Lett. 82, 2967 (1999).

[16] Handbook of Mathematical Functions, edited by M. Abramowitz and I. Stegun (Dover, New York, 1964).

[17] B. K. P. Scaife, in Complex Permittivity (The English Universities Press, London, 1971).

[18] J. H. Van Vleck et al., Rev. Mod. Phys. 17, 227 (1945).

[19] G. E. Uhlenbeck and L. S. Ornstein, Phys. Rev. 36, 823 (1930).

[20] W. T. Coffey, J. Chem. Phys. 107, 4960 (1997). 